\title{
Mars Dust Counter
}

Eduard Igenbergs ${ }^{1}$, Sho Sasaki ${ }^{2}$, Ralf Münzenmayer ${ }^{1}$, Hideo Ohashi ${ }^{3}$, Georg Färber ${ }^{4}$, Franz Fischer ${ }^{4}$, Akira Fujiwara ${ }^{5}$, Albrecht Glasmachers ${ }^{6}$, Eberhard Grün ${ }^{7}$, Yoshimi Hamabe ${ }^{2}$, Heinrich Iglseder ${ }^{8}$, Dieter Klinge ${ }^{9}$, Hideaki Miyamoto ${ }^{2}$, Tadashi Mukai ${ }^{10}$, Walter Naumann ${ }^{1}$, Ken-ichi Nogami ${ }^{11}$, Gerhard Schwehm ${ }^{9}$, Håkan Svedhem ${ }^{9}$, and Kazuo Yamakoshi ${ }^{12 *}$

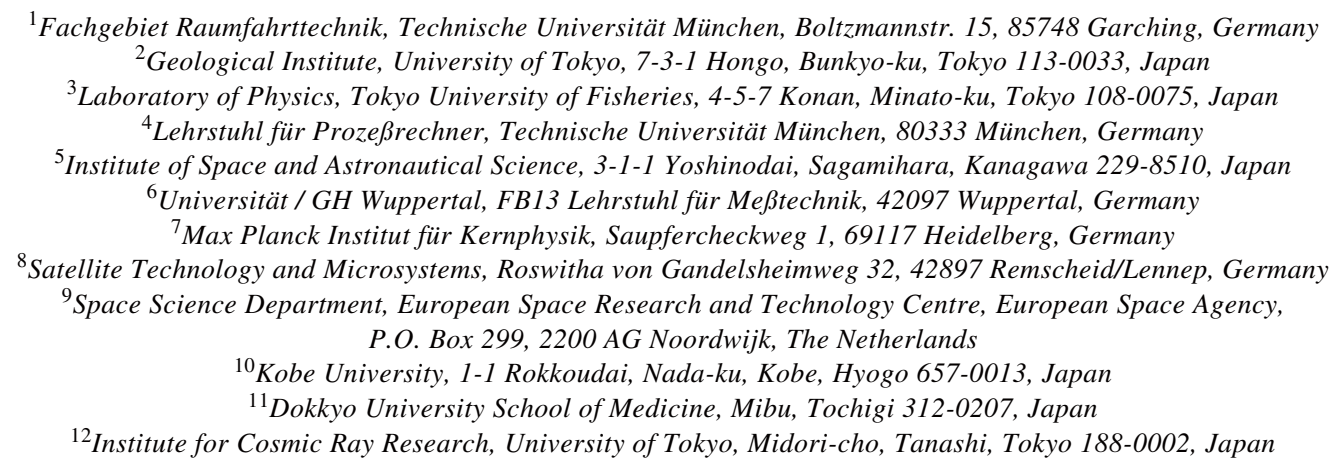

(Received July 31, 1997; Revised November 30, 1997; Accepted January 23, 1998)

In order to unveil the presence and characteristics of Martian dust ring/torus, Mars Dust Counter (MDC) is aboard ISAS's spacecraft PLANET-B, which will be launched in 1998 summer and investigate the upper atmosphere and surrounding environment of Mars between 1999 and 2001. MDC PLANET-B is an improved version of impactionization dust detectors aboard HITEN and BREMSAT. It weighs only $730 \mathrm{~g}$ with the sensor aperture area of 140 $\mathrm{cm}^{2}$. To improve signal to noise ratios and to precisely determine the risetime of signals, a neutral target channel is added independent of ion and electron target channels. Detectable velocity $(v)$ range is between $1 \mathrm{~km} / \mathrm{s}$ and more than $70 \mathrm{~km} / \mathrm{s}$, which will cover all possible dust clans: circummartian (low $v$ ), interplanetary (mid $v$ ), and interstellar $\left(\right.$ high $v$ ) particles. Measurable mass range is $5 \times 10^{-15}$ and $10^{-10} \mathrm{~g}$ at $v=10 \mathrm{~km} / \mathrm{s}$. Since PLANET-B takes an elliptic retrograde orbit around Mars, MDC can investigate particles from Phobos and Deimos with relative velocity higher than $1 \mathrm{~km} / \mathrm{s}$. Therefore, MDC can clarify the presence of a confined dust ring along Phobos' orbit and an extended dust torus along Deimos' orbit, and it may answer whether these ring and torus are self-sustained or not. Since the nominal operation of PLANET-B is longer than one Martian year, MDC may detect predicted seasonal variation of the ring/torus structure.

\section{Introduction}

Mars Dust Counter (MDC) is a light-weight impactionization type dust detector, which determines mass and velocity of a particle using time variation of generated charges (Fig. 1). MDC will be launched toward Mars aboard PLANET-B spacecraft of ISAS in July, 1998. PLANET-B will take elongated orbits whose apoapsis $\left(\sim 15 R_{\mathrm{M}} ; R_{\mathrm{M}}\right.$ being Martian equatorial radius) is far beyond the Deimos' orbit. The main objective of the mission is to study Martian aeronomy, especially interaction between the Martian upper atmosphere and the solar wind. The primary purpose of MDC is to measure dust particles around Mars and reveal distribution of the predicted Martian ring or torus of dust particles from Phobos and Deimos (Soter, 1971; Horányi et al., 1990; Ip and Banaszkiewicz, 1990; Juhász et al., 1993; Sasaki, 1993, 1994, 1996; Ishimoto and Mukai, 1994; Krivov,

\footnotetext{
*Deceased
}

Copy right () The Society of Geomagnetism and Earth, Planetary and Space Sciences (SGEPSS); The Seismological Society of Japan; The Volcanological Society of Japan; The Geodetic Society of Japan; The Japanese Society for Planetary Sciences.
1994; Juhász and Horányi, 1995; Hamilton, 1996; Ishimoto, 1996; Krivov and Hamilton, 1997). MDC can also measure other particles such as interplanetary dust, interstellar dust, and space debris around the Earth.

\section{Observation Sequences}

Observation of MDC PLANET-B is divided into three sequences according to PLANET-B orbital plans: (i) the first five-month long parking orbits around the Earth, (ii) the ten-month long transfer orbit from the Earth to Mars, and (iii) the orbits around Mars (more than 2 years).

(i) The first parking orbits are elliptic and they involve two lunar encounters. Their apogees are between 410,000 and 430,000 km and perigee altitudes are between 600 and $1200 \mathrm{~km}$ (ISAS and NEC, 1995). The final orbit between two lunar encounters is a very elliptic one whose apogee is $1,710,000 \mathrm{~km}$. Then MDC will detect interplanetary, interstellar and circumterrestrial (space debris and lunar-origin) dust particles. Other than space debris which will be detected around the low altitude and the geosynchronous orbit, lunarorigin particles which should be secondary ejecta of impacts would be detected at larger distance from the Earth. It would 


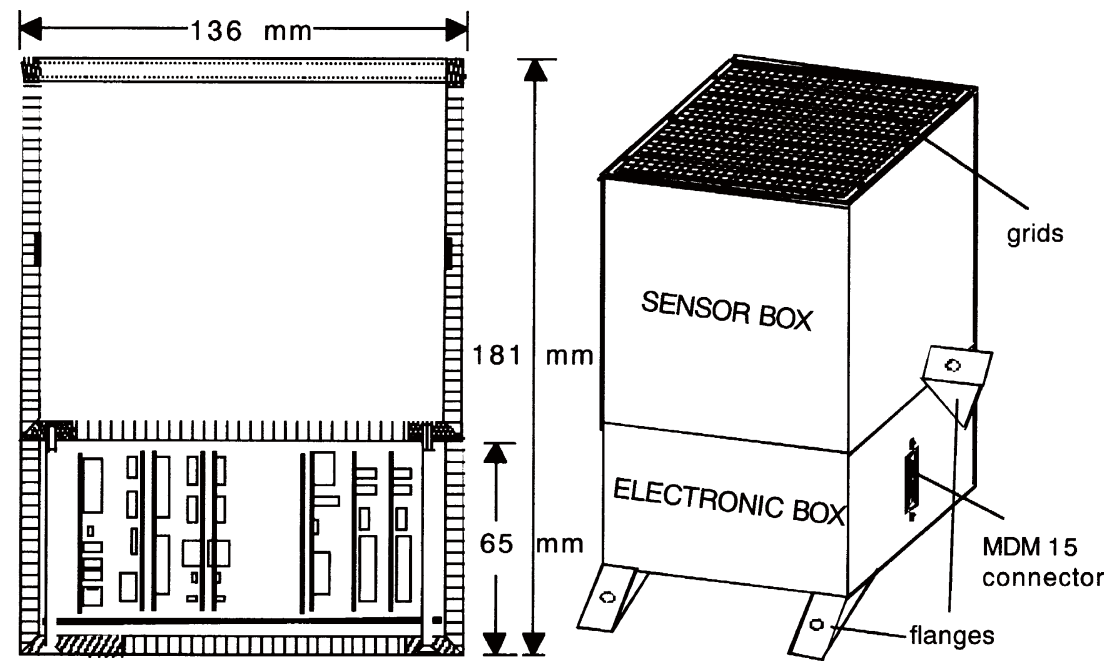

Fig. 1. The outline and the interior of MDC PLANET-B. MDC consists of the lower electronics box and the upper sensor box made of aluminum and Nomex honeycomb. All five inner walls of the sensor box are plated in gold and act as target area. The left figure shows the interior seen from the side of MDC 15 connector. There are two entrance grids which should shield electric fields. MDC is attached to one of side panels of PLANETB by four flanges.

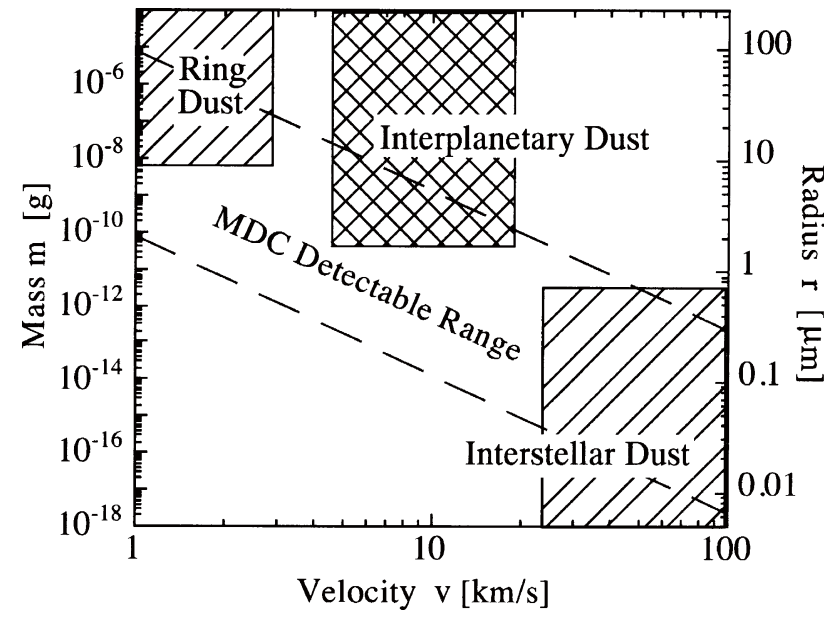

(a)

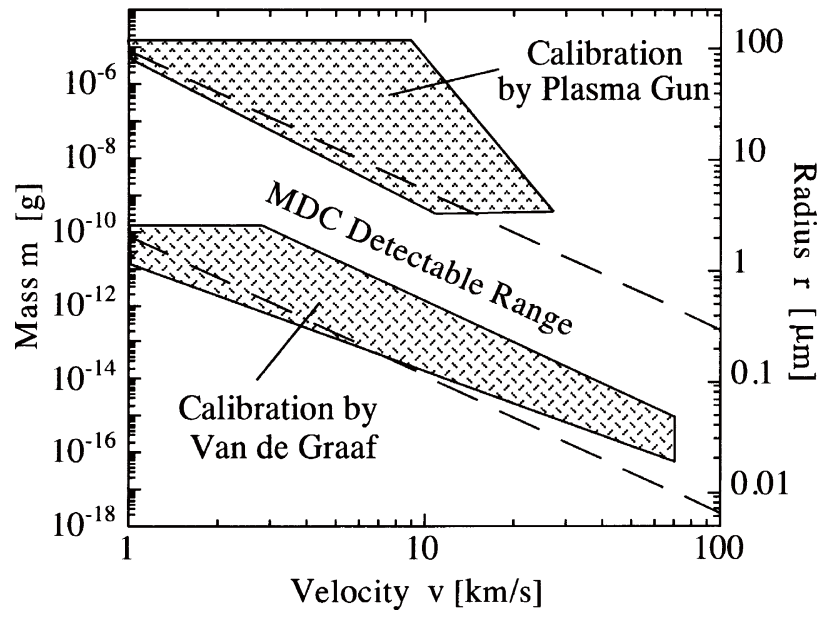

(b)

Fig. 2. Sensitivity range of MDC PLANET-B. (a) MDC sensitivity range is compared with typical ranges of relative velocity and mass of ring, interplanetary, and interstellar dust particles. (b) MDC sensitivity range is compared with capabilities of dust accelerators used for calibration. In both figures, the sensitivity range is shown between two dashed lines in velocity-mass diagram. Particles heavier than the upper limit are detected, of course, although charge saturation at collectors would make it difficult to quantitatively determine their mass. As for the radius calculation, particle density $2 \mathrm{~g} / \mathrm{cm}^{3}$ is assumed.

be unlikely to detect circumlunar dust or levitated dust from the lunar surface at the two lunar encounters, because their encounter distances from the lunar gravity center are 6255 and $4457 \mathrm{~km}$ (ISAS and NEC, 1995). Comparison of the results with previous data, especially of ISAS technologytesting spacecraft HITEN, should be useful for calibration of MDC itself. A dust detector aboard HITEN, which observed dust environment of the Earth-Moon zone for three years, was a prototype of the present MDC-PLANETB.

(ii) During the transfer orbit, MDC will measure dust particles both of interplanetary and interstellar origins. Interplanetary dust particles are supplied by asteroids and comets; they take Keplerian orbits and their orbital radii gradually decrease owing to the Poynting-Robertson effect. Their typical size is 1 to a few 10 micron $\left(10^{-11}\right.$ to $\left.10^{-7} \mathrm{~g}\right)$ and detection velocity is a few to $10 \mathrm{~km} / \mathrm{s}$. On the other hand, interstellar particles enter the solar system at relative velocity about $30 \mathrm{~km} / \mathrm{s}$ (i.e., relative velocity of the solar system with respect to surrounding interstellar gas) and their mass is less than $10^{-12} \mathrm{~g}$ (Grün et al., 1994). Then from the differences of velocity, direction, and mass, we can discriminate between interplanetary and interstellar particles (Fig. 2(a)). Ulysses and HITEN results suggest that interstellar particles entered into the inner solar system (Baguhl et al., 1996; Svedhem et al., 1996). Cassini spacecraft will go through the Earth-Mars region at the same time; MDC data can also be compared with Cassini Dust Analyzer data. 
(iii) Around Mars, PLANET-B takes elliptic retrograde orbits whose periapsis is $150 \mathrm{~km}$ from the surface and apoapsis is $15 R_{\mathrm{M}}$. Since the planned orbits are close to the zodiacal plane, they can sometimes intersect with Phobos and Deimos orbits. Because of the large apoapsis, PLANET$\mathrm{B}$ can fully encompass the expected distribution (ring/torus) of dust particles from Phobos and Deimos. Another important advantage is that relative velocity between prograde ring dust and retrograde PLANET-B will be larger than $1 \mathrm{~km} / \mathrm{s}$, which is favorable for dust measurements by an impactionization type detector. Other than circummartian particles whose velocity is less than a few $\mathrm{km} / \mathrm{s}$ and mass is larger than $10^{-9} \mathrm{~g}$, MDC will also continue measuring interplanetary and interstellar particles. Those particles can be easily distinguished from ring particles using differences of velocity, direction, and mass, as noted in the above (Fig. 2(a)). Since periapsis of PLANET-B is as low as $150 \mathrm{~km}$, we can monitor levitated dust from the lower atmosphere even if dust transport through very thin outer atmosphere would not be plausible.

\section{MDC Instrumentation}

MDC (Mars Dust Counter) is a light-weight impact ionization dust detector, which is developed chiefly by Technische Universität München (Technical University of Munich) and Space Science Department of ESA-ESTEC (European Space Research and Technology Centre of European Space Agency) (Igenbergs et al., 1996a). It is an improved version of HITEN and BREMSAT dust detectors which successfully measured dust particles around the EarthMoon region and at the low Earth orbit, respectively (Igenbergs et al., 1991a, b). The MDC was designed to determine not only flux but also mass and velocity of dust particles in space by measuring ion and electron charges produced by high velocity $(v>1 \mathrm{~km} / \mathrm{s})$ impacts of those particles on gold plate targets. Unlike other impact ionization type detectors, MDC weighs only $730 \mathrm{~g}$. Its dimension is $136 \times 127 \times 181 \mathrm{~mm}^{3}$ and sensor aperture is $124 \times 115 \mathrm{~mm}^{2}$ (Fig. 1). The power consumption of MDC is $3.78 \mathrm{~W}$.

The interior structure and mechanical outline of MDC are shown in Fig. 1. MDC consists of electronic and sensor boxes which are made of lightweight honeycomb of aluminum and Nomex. The aperture of the sensor box is covered by two sets of grounded steel grids in order to reduce electromagnetic noises from the outside and to shield internal electric field. All five inner walls, which are covered with gold plates, act as the target area for dust impacts. MDC is mounted with four flanges onto the side panel of PLANETB. The sensor aperture is looking toward 45 degree backwards from the spacecraft's spin axis, around which it scans within the field of view. Because the spin axis is parallel to the sun direction in the circumterrestrial orbit and to the Earth direction during transfer and circummartian orbits, the sensor box can avoid sunlight illumination, which would raise noise levels owing to photoelectrons.

Figure 3 shows the principle of MDC experiment. There are two charge collector plates which are biased by positive and negative voltages $( \pm 240 \mathrm{~V})$. When a dust particle impacts on the gold target, impact plasma is generated and separated into positive ions on the negative collector (ion channel) and electrons on the positive collector (electron channel). Then, impact charges are recorded by both channels. Calibration experiments suggested that gold target as well as (even rather than) dust materials should supply

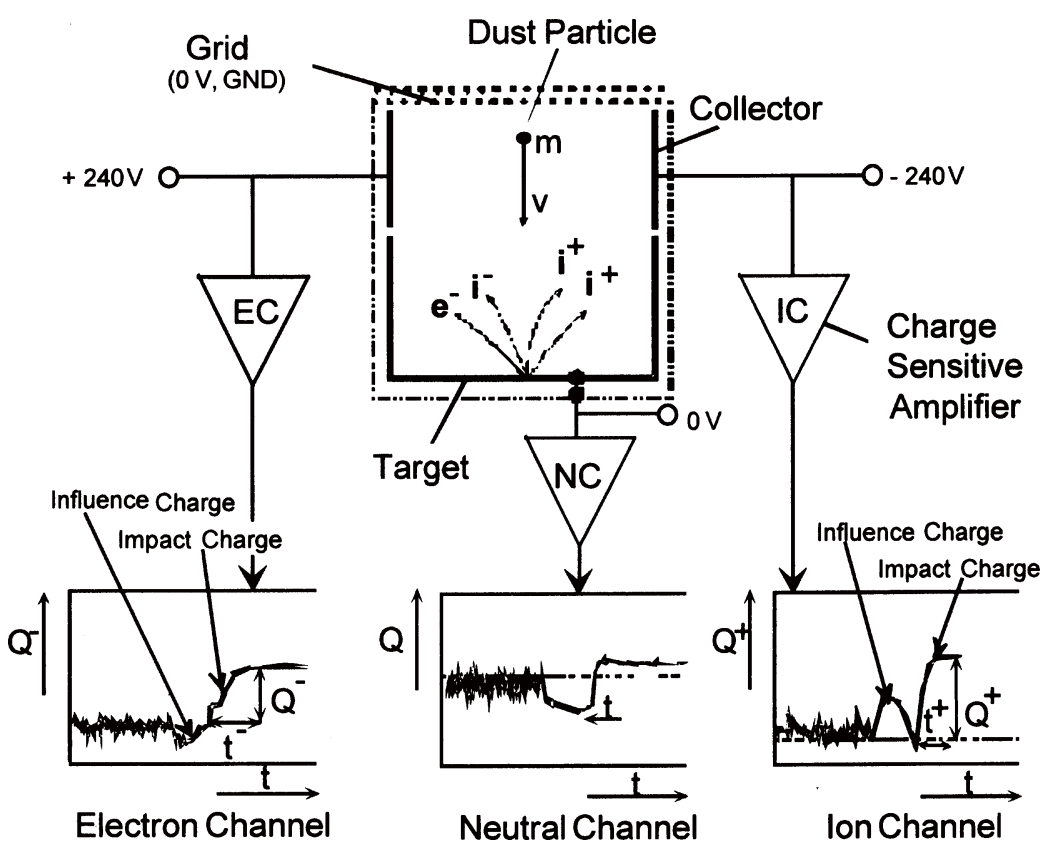

Fig. 3. Measurement principle of MDC. An impact of a dust particle on the target plate generates impact plasma. Positive ions are trapped by a negatively biased collector (ion channel) and electrons are trapped by a positively biased collector (electron channel), then generated charge signals are amplified and converted into digital data. From risetime $(t)$ and peak charge $(Q)$, information of velocity and mass of the particle is obtained. Other than positive and negative collectors, charge variation at the grounded target plates is also measured as the third channel. Then the difference of startup times gives additional information on impact velocity. 
plasma at the impact. In MDC PLANET-B, charge signals on the grounded neutral target are also recorded. This third channel is useful in distinguishing impact signals from noise signals, because in previous experiments there were events where only the electron channel had a signal and some of them could be a noise rather than a real impact. The neutral channel is also useful in determining impact velocity since we can measure time difference between the impact on the neutral target and the arrival of ions and electrons on biased plates.

The charge outputs are digitized by a transient recorder with two 8 bit A/D converters of $2.5 \mathrm{MHz}$ and a FIFO memory. To reduce error in converting charges with more than $10^{4}$ order of magnitude range to digital data, we use logarithmic charge sensitive amplifiers. Signal processing and control of spacecraft interface are done by $80 \mathrm{C} 85 \mathrm{CPU}$ with $4 \mathrm{~KB}$ ROM and $56 \mathrm{~KB}$ RAM. Each impact data is $1 \mathrm{~KB}$ with $200 \mu$ s measurement time. Up to 51 sets of impact signals can be stored in on-board RAM. Those data are transmitted to the Earth when impact number exceeds a certain warning value (e.g., 40).

In evaluating each charge signal curve, amplitude and risetime of charge are the most important values, which give mass $m$ and velocity $v$ of a dust particle. There are empirical relations

$$
\begin{aligned}
& t=c_{g} v^{\eta} \\
& \pm Q / m=c_{r} v^{\beta}
\end{aligned}
$$

where $Q$ is the maximum charge and $t$ is risetime of a charge signal. Both $Q$ and $t$ can be estimated directly from the impact charge signals as seen in Fig. 3. In the above, $c_{g}, c_{r}$, $\eta$, and $\beta$ are constants which should be determined by calibration experiments using dust accelerators (Igenbergs et al., 1996b). There are three sets of equations corresponding to electron, ion, and neutral channels. At first, using the risetime, impact velocity is estimated from Eq. (1), and then particle mass is derived from Eq. (2). As for electron and ion channels, results of calibration experiments can be expressed by the above equations for wide range of velocity between 2 and $70 \mathrm{~km} / \mathrm{s}$ (Igenbergs et al., 1996b). We have confirmed that there might be some other methods to determine velocity, e.g., using the ratio of charges due to primary impact and secondary ejecta, or using time delay between neutral, electron and ion channels, although further calibration experiments are necessary for establishing these methods.

The charge sensitive amplifiers can measure charges between $5 \times 10^{-16}$ and $1 \times 10^{-11} \mathrm{C}$. From calibration experiments, $Q / m$ at $v=10 \mathrm{~km} / \mathrm{s}$ is between 0.1 and $1 \mathrm{C} / \mathrm{g}$ depending on the impact position in the sensor. Thus, particles with mass approximately between $5 \times 10^{-15}$ and $10^{-10} \mathrm{~g}$ can be measured. This corresponds to particle diameter range between 0.1 and 10 micron at $10 \mathrm{~km} / \mathrm{s}$. Larger ( $>10$ micron at $10 \mathrm{~km} / \mathrm{s}$ ) particles can be detected, although charge saturation at collectors would make it difficult to determine their mass. Figure 2 shows the sensitivity range of MDC. Under slower impact velocity at one to a few $\mathrm{km} / \mathrm{s}$, which is expected for circummartian dust, particles as large as 100 micron would be measured.
For the purpose of the ground calibration experiments of MDC, two dust accelerator facilities are used. One is a Van de Graaf electrostatic particle accelerator at the Max Planck Institut für Kernphysik in Heidelberg, and the other is a plasmadynamic particle accelerator at Fachgebiet Raumfahrttechnik, Technische Universität München. Velocity and mass ranges executed at both facilities are shown in Fig. 2(b). Results of preliminary calibration experiments are described in Igenbergs et al. (1996b).

\section{Detection of Martian Ring/Torus}

Soter (1971) first advocated the existence of a dust ring of secondary ejecta particles when interplanetary dust particle impacts on the Phobos surface. Viking image data suggested that there is no dust ring whose optical depth is larger than $5 \times 10^{-5}$ (Duxbury and Ocampo, 1988). It was not until PHOBOS 2 ASPERA found indirect evidence of a dust/gas torus when many works started on the subject on the Martian dust ring. There, ion mass spectrometry suggested the existence of a large mass number particles, which could be ascribed to very fine dust (<0.1 micron) (Dubinin et al., 1990).

Recent theoretical studies (e.g., Juhász and Horányi, 1995; Hamilton, 1996; Ishimoto, 1996; Sasaki, 1996) show that solar radiation pressure as well as Martian oblateness should enhance the orbital eccentricity of particles (from both Phobos and Deimos) and inclination of particles (from Deimos only) greatly. As for particles from Phobos, eccentricity of particles smaller than 200 micron is greatly increased owing to the resonance of phase shift due to Martian oblateness. And eccentricity of particles smaller than $20 \mathrm{mi}-$ cron becomes so large that they are quickly captured by Mars. Since inclination is not largely increased, dust particles from Phobos would form a thin dust ring whose thickness would be less than $300 \mathrm{~km}$. The eccentricity of dust particles from Deimos is also enhanced by radiation pressure, but the combined effect with Martian oblateness also increases inclination to be as high as 0.2 to form an extended torus, which would contain smaller particles than those of the Phobos' ring.

If the secondary ejecta only from interplanetary dust impacts on the satellite surfaces should contribute the circummartian dust, expected detection number of ring/ torus particles would be smaller than that of interplanetary particle. However, collisions of once-ejected ring particles on the satellites may produce additional dust particles, since the surfaces of Phobos and Deimos are covered with regolith and their escape velocities are as small as $10 \mathrm{~m} / \mathrm{s}$. In this selfsustained case (Sasaki, 1994, 1996), expected dust number density will be much higher so that MDC will detect the Phobos' ring and the Deimos' torus.

Hamilton (1996) and Krivov and Hamilton (1997) predict seasonal variation of dust ring structures. If dust abundance is high enough, MDC may detect change of dust distribution according to seasons, since nominal duration of PLANETB operation is one Martian year.

Although the predicted dust ring seems to be distributed uniformly in space, the detection of fresh, rather dense dust ejecta is expected near satellites. Planet-B will encounter with Phobos on about 280th and 420th days and with Deimos on about 150th and 550th days from the insertion to 
Mars. During those close encounters, MDC may measure direct dust ejecta from satellites.

Acknowledgments. We acknowledge Jose Maria Castro for the supporting work on MDC electronics, Gerhard Schäber and Ralf Srama for the assistance during calibration experiments at MPI accelerator, and Kurt Graf and Rainer Ondrusch for laboratory works at Technische Universität München. We thank Hiroshi Ishimoto and Doug Hamilton for discussions on dust rings. We are grateful to anonymous reviewers for helpful comments.

\section{References}

Baguhl, M., E. Grün, and M. Landgraf, In situ measurements of interstellar dust with the Ulysses and Galileo Spaceprobes, Space Sci. Rev., 78, 165 $172,1996$.

Dubinin, E. M., R. Lundin, N. F. Pissarenko, S. V. Barabash, A. V. Zakharov, H. Koskinen, K. Schwingenshuh, and Y. G. Yeroshenko, Indirect evidences for a gas/dust torus along the Phobos orbit, Geophys. Res. Lett., 17, 861-864, 1990.

Duxbury, T. C. and A. C. Ocampo, Mars: satellite and ring search from Vikings, Icarus, 76, 160-162, 1988.

Grün, E., B. Gustafson, I. Mann, M. Baguhl, G. E. Morfill, P. Staubach, A. Taylor, and H. A. Zook, Interstellar dust in the heliosphere, Astron. Astrophys., 286, 915-924, 1994.

Hamilton, D. P., The asymmetric time-variable rings of Mars, Icarus, 119 , 153-172, 1996.

Horányi, M., J. A. Burns, M. Tátrallyay, and J. G. Luhmann, Toward understanding the fate of dust lost from the Martian satellites, Geophys. Res. Lett., 17, 853-856, 1990.

Igenbergs, E., A. Hüdepohl, K. Uesugi, T. Hayashi, H. Svedhem, H. Iglseder, G. Koller, A. Glasmachers, E. Grün, G. Schwehm, H. Mizutani, T. Yamamoto, A. Fujimura, N. Ishii, H. Araki, K. Yamakoshi, and K. Nogami, The present status of the Munich Dust Counter experiment on board of the HITEN spacecraft, in Origin and Evolution of Interplanetary Dust, edited by A. C. Levasseur-Regourd and H. Hasegawa, pp. 15-20, Kluwer, 1991a.

Igenbergs, E., A. Hüdepohl, K. Uesugi, T. Hayashi, H. Svedhem, H. Iglseder, G. Koller, A. Glasmachers, E. Grün, G. Schwehm, H. Mizutani, T. Yamamoto, A. Fujimura, N. Ishii, H. Araki, K. Yamakoshi, and K. Nogami, The Munich dust counter: A cosmic dust experiment on board of the MUSES-A mission of Japan, in Origin and Evolution of Interplanetary Dust, edited by A. C. Levasseur-Regourd and H. Hasegawa, pp. 45-48, Kluwer, 1991b.

Igenbergs, E., S. Sasaki, G. Fäber, F. Fischer, R. Münzenmayer, A. Fujiwara, H. Iglseder, A. Glasmachers, E. Grün, T. Mukai, H. Ohashi, G. Schwehm, H. Svedhem, and K. Yamakoshi, Mars Dust Counter on Board ISAS PLANET-B, in Physics, Chemistry, and Dynamics of Interplanetary Dust, edited by B. A. S. Gustafson and M. S. Hanner,
ASP Conf. Ser., 104, 237-340, 1996a.

Igenbergs, E., R. Münzenmayer, S. Sasaki, G. Fäber, F. Fischer, A. Fujiwara, H. Iglseder, A. Glasmachers, E. Grün, T. Mukai, H. Ohashi, G. Schwehm, H. Svedhem, and K. Yamakoshi, Mars Dust Counter on Board ISAS PLANET-B, in Proceedings of 20th International Symposium on Space Technology and Space, pp. 1222-1229, Gifu, Japan, $1996 \mathrm{~b}$.

Ip, W.-H. and M. Banaszkiewicz, On the dust/gas tori of Phobos and Deimos, Geophys. Res. Lett., 17, 857-860, 1990.

ISAS and NEC, Orbit Design in PLANET-B Interim Report, SES-TD-94022, Institute of Space and Astronautical Science SES Data Center, 1995.

Ishimoto, H., Formation of Phobos/Deimos dust rings, Icarus, 122, 153$165,1996$.

Ishimoto, H. and T. Mukai, Phobos dust rings, Planet. Space Sci., 42, 691697, 1994.

Juhász, A. and M. Horányi, Dust torus around Mars, J. Geophys. Res., 100, 3277-3284, 1995.

Juhász, A., M. Tatrállyay, G. Gévai, and M. Horányi, On the density of the dust halo around Mars, J. Geophys. Res., 98, 1205-1211, 1993.

Krivov, A. V., On the dust belts of Mars, Astron. Astrophys., 291, 657663, 1994.

Krivov, A. V. and D. P. Hamilton, Martian dust belts: waiting for discovery, Icarus, 128, 335-353, 1997.

Sasaki, S., Evolution of dust particle tori around Mars, in Proc. 26th ISAS Lunar Planet. Symp., edited by M. Shimizu and H. Mizutani, pp. 4043, 1993.

Sasaki, S., Martian dust tori formation: Ejecta at collision of torus particles with the satellite can sustain dust abundance, in Proc. 27th ISAS Lunar Planet. Symp., edited by M. Shimizu and H. Mizutani, pp. 47-50, 1994.

Sasaki, S., Martian self-sustaining dust torus, in Physics, Chemistry, and Dynamics of Interplanetary Dust, edited by B. A. S. Gustafson and M. S. Hanner, ASP Conf. Ser., 104, 187-190, 1996.

Soter, S., The dust belts of Mars, Report of Center for Radiophysics and Space Research 462, Cornell Univ., Ithaca, 1971.

Svedhem, H., R. Münzenmayer, and H. Iglseder, Detection of possible interstellar particles by the HITEN spacecraft, in Physics, Chemistry, and Dynamics of Interplanetary Dust, edited by B. A. S. Gustafson and M. S. Hanner, ASP Conf. Ser., 104, 27-30, 1996.

E. Igenbergs (e-mail: E.Igenbergs@1rt.mw.tu-muenchen.de), S. Sasaki (e-mail: sho@geol.s.u-tokyo.ac.jp), R. Münzenmayer (e-mail: R.Muenzenmayer@lrt.mw.tu-muenchen.de), H. Ohashi (e-mail: ohashi@tokyo-u-fish.ac.jp), G. Färber, F. Fischer, A. Fujiwara, A. Glasmachers, E. Grün, Y. Hamabe, H. Iglseder, D. Klinge, H. Miyamoto, T. Mukai, W. Naumann, K. Nogami, G. Schwehm, H. Svedhem, and K. Yamakoshi 